\title{
Good Enough Yet? A Preliminary Evaluation of Human-Surrogate Interaction
}

\author{
Julian Abich IV ${ }^{1}$, Lauren E. Reinerman-Jones ${ }^{1}$, Gerald Matthews ${ }^{1}$, \\ Gregory F. Welch ${ }^{2}$, Stephanie J. Lackey ${ }^{1}$, Charles E. Hughes ${ }^{2}$, and Arjun Nagendran ${ }^{2}$ \\ ${ }^{1}$ University of Central Florida (UCF), Institute for Simulation \& Training (IST), \\ Applied Cognition in Virtual Immersive Training Environments Laboratory (ACTIVE Lab) \\ Orlando, FL, USA \\ \{jabich, lreinerm, gmatthew, slackey\}@ist.ucf.edu \\ ${ }^{2}$ University of Central Florida (UCF), Institute for Simulation \& Training (IST), \\ Synthetic Reality Lab (SREAL) \\ welch@ucf.edu, \{ceh,arjun\}@cs.ucf.edu
}

\begin{abstract}
Research exploring the implementation of surrogates has included areas such as training (Chuah et al., 2013), education (Yamashita, Kuzuoka, Fujimon, \& Hirose, 2007), and entertainment (Boberg, Piippo, \& Ollila, 2008). Determining the characteristics of the surrogate that could potentially influence the human's behavioral responses during human-surrogate interactions is of importance. The present work will draw on the literature about human-robot interaction (HRI), social psychology literature regarding the impact that the presence of a surrogate has on another human, and communications literature about human-human interpersonal interaction. The review will result in an experimental design to evaluate various dimensions of the space of humansurrogate characteristics influence on interaction.
\end{abstract}

Keywords: human-robot interaction, human-surrogate interaction, communications, social psychology, avatar, physical-virtual avatar.

\section{Introduction}

The past decades have seen a tremendous social role change for robots and virtual avatars, as many have forecasted (Dragone et al., 2006). One such manifestation is the inception of their use as non-traditional surrogates (Welch, 2012). Surrogates, although the term has other meanings (McFarland et al., 2002), are considered for the present purpose to be a substitute for another person in a context-specific role. These surrogates can range from being purely virtual characters to real human beings. In the broadest sense, the term 'surrogate' captures the interest in human representations, while not being encumbered by traditional distinctions between digital and physical form (of the virtual characters) or the nature of the agency (intelligence model of the virtual characters). Basically, the concentration here is on the 'vessel' that will house the surrogate and to describe how to conceptualize the future social roles of nontraditional surrogates. 
As the average person in many industrialized nations becomes more tech savvy, they are less likely to perceive interactions with robots as negative (Bartneck et al., 2005). The substantial increase in computer-mediated communication (CMC) is a factor in this acceptance (Halpern \& Katz, 2013). Haraway (1985) even made arguments stating the social response humans have towards robots can be attributed to the societal trend of blending computers, machines, and organisms. This has created an environment in which people are more willing to at least entertain the idea of interacting with surrogates in the forms of robots and virtual avatars. Reeves and Nass (1996) also found that humans are likely to socially accept computer entities as equal peers as long as they consistently portray competent social behavior. Therefore, on a larger scale, research should seek the benefits of implementation of non-traditional surrogates for current social roles held by a human. Before this accomplishment, basic lines of research revealing the perception of the human user should lay foundational work and provide recommendations for non-traditional surrogate design and application. Despite the acceptance of these newly acquired avenues of interaction, little is known about the factors that influence the behavioral response from the human user. Specifically, it is unknown how the perceived interaction of the human user will be affected by the characteristics of these non-traditional surrogates and if the same forms of communication that apply to human-human interactions still hold true for human-surrogate interaction, in all its forms (Welch, 2012).

An initial review of the current state of human-surrogate interaction is presented. The work will then draw on the literature about human-robot interaction (HRI). However, it should be noted in relation to the developmental progress of physical-virtual avatars, that much of the work has centered on improving the functionality and capabilities of the robot to resemble a human, such as the motion and behavior, but fewer efforts have focused on the appearance (Lincoln et al., 2010) or the human's perception of the robotic entities. To that end, best practices for experimentation will also draw on the social psychology literature regarding the impact that the presence (actual, imagined, or implied) of a surrogate has on another human (Fiske, 2004) and the communications literature about human-human interpersonal interaction.

The first step in this line of research is understanding each of these disciplines separately and then integrating the concepts into a shared mental model from which surrogate research can flow. This process, in particular identifying specific constructs, will be highlighted in the present paper. The evaluation of various approaches to studying similar domain areas and their importance to driving novel surrogate research will be discussed in more detail. The result of this review is an initial experimental design constructed from a truly integrated, multi-disciplinary viewpoint. That experimental design will be outlined with the expected impact on a newly developed systematic approach to studying human-surrogate interactions.

\subsection{Human-Surrogate Interaction}

Traditionally, human surrogates have played a variety of roles within social settings, such as medical, military, educational, and entertainment. Waytz (2013) argues that meaningful connections with "social substitutes" are possible. Even in other species, 
surrogates have been shown to elicit a social response (Harlow, 1958). There is an apparent innate desire to develop social ties. Some might argue that from an evolutionary perspective, successful socialization increases the likelihood of survival and reproduction. Therefore, one must take advantage of this motivation to socialize when developing the role of a non-traditional surrogate.

Advances in computer science technology have led to the development of many forms of surrogates, including virtual and physical-virtual. The vision of future surrogate roles is one of a widespread generality. Imagine classrooms all over the world that are each equipped with a human-like surrogate that receives input from a single teacher and portrays her dynamic image from a remote location (Welch, 2012). This allows people to be in more than one place at a time, disseminating thoughts, ideas, and knowledge to a larger population. Now replace that teacher with a doctor, and now the doctor has the ability to visit with patients, nurses, or other colleagues without having to be physically present (Lincoln et al., 2010). Use the role of a search and rescue team instead, and now teams can access areas that are potentially dangerous or impenetrable for a human, but not for a surrogate (Goodrich \& Schultz, 2007). As one can see, this opens up the door to new ways of interacting and communicating, expanding the physical and mental capabilities of both surrogates and users. Research has explored the successful implementation of non-traditional surrogates in areas such as training (Chuah et al., 2013), education (Yamashita, Kuzuoka, Fujimon, \& Hirose, 2007), and entertainment (Boberg, Piippo, \& Ollila, 2008), yet the field is still in its infancy.

One type of possible physical-virtual surrogate is a robotic replica of a person (Lincoln et al., 2010; Welch, 2012). Determining the characteristics of the surrogate that could potentially influence the human's behavioral responses during humansurrogate interactions is of importance for effective interactions. It would be advantageous for these robotic replicas to capitalize on the extensive research within the field of HRI to aid non-traditional surrogate development.

\subsection{Human-Robot Interaction}

HRI is the "field of study dedicated to understanding, designing, and evaluating robotic systems for use by or with humans" (Goodrich \& Schultz, 2007). The interaction, therefore, necessitates some form of communication to occur between both the human and robot. Several forms of communications are possible, but are dependent upon proximity to each other (Mumm \& Mutlu, 2011). Entities can either be distant or nearby. In reference to human-surrogate interaction, research should compare the effectiveness of interacting and communicating with surrogates that are remote or co-located in a variety of contexts to establish when it is appropriate to use one or the other.

Goodrich \& Shultz (2007) described five attributes in which interaction between a human and robot is possible. Of the five, two are most relevant to the current review. One centers on the nature of information exchange and the other, of a lesser immediate relevance, refers to the adaptation, learning and training of humans and robots. Many of the characteristics of the former attribute indicates the way information is 
passed, such as the format, medium, or rules (Chan et al., 2005; Gunhee Kim et al., 2004; Sidner et al., 2004), and reaches out to the human factors and communication literature for support. HRI addresses, in this context, the parameters of the information need to be in order for the human perceptual system to effectively receive such input. The ability to satisfactorily understand, communicate, or interpret the behavior of a robot or avatar will factor into the perceived usability of interacting with the surrogate (Yanco, Drury, \& Scholtz, 2004). The second attribute takes two approaches, maximizing or minimizing the amount of training required to effectively interact with the robot. For naturalistic interactions and increased likelihood of technological acceptance, minimization is preferable (Salvini, Laschi, \& Dario, 2010).

From a once common teleoperated or supervisory control, these entities have acquired more autonomous capabilities to allow free roaming interaction in the social environment with other robots, computers, humans, or a combination thereof. If surrogates are going to be used for a myriad of social roles, then ideally, extensive training should not be required to learn how to communicate with each other. They should both hold a similar mental model as to how the interaction or communication should take place (Crandall \& Goodrich, 2002). By leveraging the anthropomorphic tendency of humans to ascribe human characteristics to animals and objects, mental models of human-human interactions will transfer to a human-robot interaction, facilitating the transition of utilizing a robot or virtual avatar as a medium through which surrogates are portrayed (Halpern \& Katz, 2013). A look at the role of human perception as a motivational factor for socializing with surrogates borrows explanations from the discipline of social psychology.

\subsection{Social Psychology}

Social Psychology refers to the "scientific attempt to understand and explain how the thoughts, feelings, and behaviors of individuals are influenced by the actual, imagined, or implied presence of other human beings" (Fiske, 2002, 2004; Stevens \& Fiske, 1995). Presence is the key word here. Social psychology deals with how individuals think and behave when in the presence of others. Research showed that a physically present robot was associated with changes in participant engagement when compared to a video image of the same robot, suggesting that physical presence is an influential factor (Welch, 2012). What is unknown is the context or situation in which the physical or imagined presence of a surrogate is most effective for human-surrogate interaction.

Individuals can express themselves in ways that can influence a social group and vice versa. If certain criteria are met, such as perceived benefits or trust, individuals within the social group develop interpersonal relationships, increasing dependence on each other (Yuan, Fulk, Monge, \& Contractor, 2010). This increase leads to interdependence of individuals working together to accomplish a shared goal because of the perceived benefits they will gain by a collective effort, rather than individual. Therefore, for successful instantiation of non-traditional surrogates, interdependence is crucial. To increase the success rate of interdependence, it is valuable to identify and understand the motivation for individuals to socialize. 
The core social motives framework describe by Fiske (2004) attempts to describe the psychological processing that underlies human cognition, affect, and behavior within a social context. One core social motive, belonging, underlies the other four motives, which include controlling, understanding, self-enhancing, and trusting. For example, in applied settings, it may be useful to foster a sense that humans and robots 'belong to the same team.' Social thoughts, feelings, and behaviors are argued to be linked to satisfying these core social motives. In terms of human-surrogate interaction, the relationship built between the two should aim to meet these motives. By securing these motives, a shared goal can be met and the level (i.e. strength, frequency, diversity) of interdependence will increase (Fiske, 2004; Yuan, Fulk, Monge, \& Contractor, 2010). There are also many individual factors that can affect the way in which humans socialize, such as culture (Bartneck et al., 2005), gender (Nomura \& Takagi, 2011; Schermerhorn, Scheutz, \& Crowell, 2008), and religion (ShawGarlock, 2009), and must be taken in account when defining the context in which human-surrogate interaction will occur. The context will suggest the way in which communication will ensue during human-surrogate interaction.

\subsection{Communication}

Communicating is an act of transmitting verbal and non-verbal information (Lackey et al., 2013). Transmission refers to a message being communicated from one entity to another (i.e. asking a question). Communication entails a bi-directional passage of information between two entities through a common system (i.e. symbols, signs, behavior). Human communication is comprised of more than just the information conveyed, but a composition of psychological, physiological, behavioral, and environmental factors (Floyd \& Afifi, 2011), with goals to establish/maintain a relationship, persuade changes, understand others, and/or reach a common goal. When applying these concepts to the human-surrogate interaction domain, the content of the message is not conveyed alone, but a synthesis of explicit and implicit information in communication forms that fluctuate depending on the content and context of the message.

Of the forms of human communication, the most relevant to construct positive human-surrogate interactions are a mix of interpersonal, interviewing, and CMC. Interpersonal communication suggests a relationship exists or builds between the two interacting partners. Interviewing consists of more direct questions and answers, but still has interpersonal value. CMC is a general form in which communication is facilitated by a computer or computer-related device. By allowing the form of human-surrogate communication to vary, it will resemble the forms used during human-human communication, likely increasing the social presence of the surrogate from the perspective of the user.

Though communication seems to be expressed rather explicitly through these various forms of communication, there are a host of implicit cues that are expressed as well (Lackey et al., 2011; Reinerman-Jones, Sollins, \& Hudson, 2013). Effective communication depends not just on the clarity and relevance of verbal utterances, but also on non-verbal cues that facilitate understanding of the speaker and regulation of the dialogue (Breazeal et al., 2005). These cues include gestures and displays of emotion. Indeed, in some accounts, facilitating communication is a primary function 
of emotion (Oatley \& Johnson-Laird, 2014). To some degree, both emotional and gestural cues are processed unconsciously. Thus, an effective surrogate needs, at least to some degree, to elicit implicit processing of cues similar to human-human interaction. Ideally, the interaction feels natural to the human participant, even if he or she is unaware of the cues that facilitate communication. These concepts are theorized in a transactional model of communication.

According to the transaction model, communication is never a one-way street, but a simultaneous, mutual interaction (Barnlund, 1970; Watzlawick, Beavin, \& Jackson, 1967; West \& Turner, 2009), unlike the linear models that argue communication is more one-sided. The term transaction implies a supportive interaction to attain a collective meaning (West \& Turner, 2009). That is the goal of human-surrogate interaction, to build a trusting relationship in order to work together to develop a shared meaning of the situation and progress towards a cooperative solution.

\section{Interaction Investigation Recommendations}

The purpose of these general logic statements is to suggest the areas of investigational importance and how they relate to achieve successful human-surrogate interaction. This logic, ideally to increase the successful implementation of non-traditional surrogates, is based on the disciplines described above.

- Perception: if humans perceive the surrogate as beneficial or coherent to their mental models, then they will trust it

- Trust: if humans trust the surrogate, then they will use it

- Usability: if humans are able to use the surrogate, then they will accept it

- Technological acceptance: if humans accept the surrogate, then interactions will be effective and performance will meet or exceed set standards

- Performance: if human performance meets or exceeds standards, then humansurrogate interaction is successful

Graaf and Allouch, (2013) suggested including both hedonic and utilitarian factors to assess the social role of these surrogates. The first two relate to the hedonic factors of the user's perspective and interpersonal relationships, while the following three evaluate the utilitarian aspects of interaction and experience. A salient interpretation of the human-surrogate interaction experience will begin to emerge by looking at both factors, informing the ultimate decision to either use non-traditional surrogates or not. This is intended not as an exhaustive effort to categorize all human-surrogate interaction research areas, but as a guiding framework to add or remove logical investigative statements or sub-statements to direct human-surrogate interaction research based on contextual purposes.

\subsection{Experimental Approach}

The novel experimental approach proposed here seeks to combine the guiding theories and principles as interdisciplinary solutions to the interdisciplinary investigation 
of manifesting a surrogate from the combination of human, virtual avatar, and robotic features to effectively communicate with a human to achieve a shared goal. The term human-surrogate interaction implies the cooperation of two or more individuals, meaning no single entity (i.e. human, robot, avatar) can provide all the solutions. This consideration implicitly directs the approach that must be taken in order to address the research issues. The manifestations of virtual and physical-virtual surrogates typically are represented with many anthropomorphic features, come in a variety of shapes and physical forms, and have varying levels of intelligence, but the degree to which these features influence the human's response, and therefore potentially impact the effectiveness of communication, is less understood (Dragone et al., 2006; Halpern \& Katz, 2013; Welch, 2012). Thus, it is important to conduct controlled experimental studies of the impact of specific types of surrogate on the experience of the human who interacts with them.

\section{Experimental Overview}

Central to the methodology is the use of multiple metrics for evaluating participant experience. These metrics include both scales for explicit aspects of experience, and implicit behavioral measures. Scales for explicit response may be located within the research literatures already reviewed, i.e., those on HRI and the human factors of interfaces, on social perceptions, and on conscious perceptions of communication. Implicit behavioral measures may be derived primarily from communications research on objective measures of speech behavior and postural cues to speaker and listener engagement. Statistical analysis can then be used to determine whether and how surrogate type influences these various metrics.

\subsection{Variables}

Independent. The independent variable is surrogate type, manipulated withinsubjects, includes a human, 2D virtual avatar, and a physical-virtual avatar (PVA). All three types of surrogates should be used because the participant's experience may be so profoundly influenced by interacting with a virtual figure as opposed to a human, that the experience becomes qualitatively different. One aim is then to compare the participant's responses to human and virtual surrogates along relevant metrics to determine whether it is feasible to use responses to the human as a reference point for evaluating a range of different surrogates.

The gender, age, voice, clothing, and form of interaction should be held constant across all surrogate types, the difference being solely the medium in which the surrogate is presented. The surrogates should express the seven universal primary emotions (Ekman, 1992) through tone of voice, gestural cues, and facial expression while interacting with participants. Gestural cues (head movements) and facial emotion expressions can be programmed or practiced (depending on the surrogate type) to represent the use of cues in human-surrogate interaction. 


\section{Dependent}

Subjective Measures. Questionnaires should be based on the multidisciplinary approach described and used to gather subjective information from the participants about their experience with each surrogate. They should cover areas related to the user's perception, trust, usability, and technology acceptance. As described in section 1 above, the literature provides several relevant constructs. From a social psychological perspective, it is important also to assess the user's interpretation of their experience of the interaction with the surrogates in relation to constructs such as social presence. The assessment of trust should look at the perceptions of the reliability, functionality, and social attributes of the surrogates. As with any form of technology, the ability to effectively and efficiently use it should be addressed by a usability questionnaire. Technology acceptance refers to the interest and enjoyment of the interaction with the surrogates. Especially in the early stages of research, it is important also to obtain qualitative responses, which may illuminate data obtained from quantitative rating scales. Qualitative data may be obtained from free responses to questions probing key elements of the user experience. The free response format also allows assessment of the user's experience of communication with the surrogate, and any specific issues arising from the visual and auditory components of communication.

Objective Measures. Validated questionnaire scales may be more effective in evaluating explicit rather than implicit responses. As implicit measures, postural measures may pick up unconscious reactions that may not be evident in the questionnaires (Knapp, Hall, \& Horgan, 2013).

Postural Measures. A head-tracking system can be used to objectively assess the participant's head position and a body-tracking system can be used for assessing the torso and arms/hands of the participants while engaged in transactional conversations with the surrogates.

Verbal Communication Measures. Objective measures of the participant's verbal responses can be assessed during communication with each surrogate of interest. Measures include frequency counts of utterances, average duration, and average response time to surrogate speech.

\subsection{Procedure}

It is important to maintain experimental control of participants' interactions with the three surrogates of interests (human, virtual, and physical-virtual) through verbal communication. Both the participant and the surrogate should remain at a constant distance from each other and never be in physical contact to first assess characteristics of the surrogate without taking into account other factors such as proximity. Communication can take the form of structured verbal exchanges between the participant and the surrogate, resembling casual conversation about both non-emotional (i.e., asking the participant's age) and emotional topics (i.e. asking about emotional reactions to being cut off while driving). The suggested post-exposure questionnaires can be 
administered following interaction with each surrogate. The order of experimental conditions (exposure to each surrogate) must also be counter-balanced across participants to minimize any order effects.

\section{Conclusion}

Our proposed methodology will potentially identify characteristics of surrogates that can affect human perceptions of transactions with surrogates, achieved through a multi-disciplinary method. The multi-disciplinary approach is encouraged to explore the area of human-surrogate interaction. Specifically, in our research, we aim to compare the participant's experience of three surrogate types across a range of quantitative metrics derived from the relevant disciplines. We can then evaluate differences between surrogates in terms of constructs derived from HRI (e.g., usability), social psychology (e.g., presence) and communication (e.g., postural engagement). We anticipate finding different patterns or profiles of response to the three surrogates, patterns that can be interpreted qualitatively on the basis of the relevant research literatures, aided by the participant's free-response accounts of their experience.

Two specific types of comparison are of most important. First, we can compare participants' reactions to human versus virtual surrogates. The cultural trend towards increased interaction with artificial systems (Bartneck et al., 2005) implies that people may have broadly similar experiences of interacting with the two types of entities, at least in highly structured settings, such as that developed for this study. On the other hand, the distinction between human and virtual presence may be so salient that the experiences cannot be considered comparable. Based on the outcome of the comparison, we can explore a defined single domain for human-surrogate interaction or pursue two separate lines of investigation. That is, if there are major qualitative differences in interacting with the two forms of surrogate, it may not be productive to compare interactions with virtual surrogates (whether human or artificial) with those with physically-present human beings.

Second, within the field of virtual systems, the research may begin to inform about optimal design, for example, the auditory and visual characteristics that optimize natural interaction. The current study should be informative about one specific design question, whether to use a 2-D or 3-D figure. Questionnaires also probe some specific design issues such as the acceptability of the visual and auditory aspects of the stimuli, and the realism of the emotional displays. Future studies might explore the impact of other design features, such as proximity between the human and surrogate (Halpern \& Katz, 2013; Mumm \& Mutlu, 2011).

The applied contribution of the research will be to guide practitioners, designers, and trainers in managing some of the tradeoffs faced when creating human surrogates (Welch, 2012). It will also make a methodological contribution towards developing protocols for evaluating the person's experience with surrogates, which might be directed towards a range of surrogate systems and contexts for interaction. The findings will advance the understanding and framework of human-surrogate interaction and its social role as investigated through the use of virtual and physical surrogates. 
Acknowledgements. This work was in part supported by the Office of Naval Research (ONR) (N00014-12-1-0052). The views and conclusions contained in this document are those of the authors and should not be interpreted as representing the official policies, either expressed or implied, of ONR or the US Government. The US Government is authorized to reproduce and distribute reprints for Government purposes notwithstanding any copyright notation hereon.

\section{References}

1. Barnlund, D.C.: A transactional model of communication. Foundations of Communication Theory, 83-102 (1970)

2. Bartneck, C., Nomura, T., Kanda, T., Suzuki, T., Kato, K.: Cultural differences in attitudes toward robots. In: Proceedings of Symposium on Robot Companions: Hard Problems and Open Challenges in Robot-human Interaction (SSAISB 2005), pp. 1-4 (2005)

3. Boberg, M., Piippo, P., Ollila, E.: Designing avatars. In: Proceedings from the 3rd International Conference on Digital Interactive Media in Entertainment and Arts, pp. 232-239. ACM (2008)

4. Breazeal, C., Kidd, C.D., Thomaz, A.L., Hoffman, G., Berlin, M.: Effects of nonverbal communication on efficiency and robustness in human-robot teamwork. In: Proceedings of IEEE/RSJ International Conference on Intelligent Robots and Systems, pp. 708-713. IEEE (2005)

5. Chan, A., MacLean, K., McGrenere, J.: Learning and identifying haptic icons under workload. In: Eurohaptics Conference, 2005 and Symposium on Haptic Interfaces for Virtual Environment and Teleoperator Systems, 2005. World Haptics 2005. First Joint, pp. 432439. IEEE (2005)

6. Chuah, J.H., Robb, A., White, C., Wendling, A., Lampotang, S., Kopper, R., Lok, B.: Exploring agent physicality and social presence for medical team training. Presence: Teleoperators and Virtual Environments 22, 141-170 (2013)

7. Crandall, J.W., Goodrich, M.A.: Characterizing efficiency of human robot interaction: A case study of shared-control teleoperation. In: Proceedings from International Conference on Intelligent Robots and Systems, vol. 2, pp. 1290-1295. IEEE (2002)

8. Dragone, M., Duffy, B.R., Holz, T., O' Hare, G.M.P.: Fusing realities in human-robot social interaction. VDI BERICHTE 1956, 23 (2006)

9. Ekman, P.: Are there basic emotions? Psychological Review 99(3), 550-553 (1992)

10. Floyd, K., Afifi, T.D.: Biological and physiological perspectives on interpersonal communication. The SAGE Handbook of Interpersonal Communication 87 (2011)

11. Fiske, S.T.: Five core social motives, plus or minus five. In: Spencer, S.J., Fein, S., Zanna, M.P., Olson, J. (eds.) Motivated Social Perception: The Ontario Symposium, pp. 233-246. Erlbaum, Mahwah (2002)

12. Fiske, S.T.: Social beings: A core motives approach to social psychology. John Wiley \& Sons, Hoboken (2004)

13. Garau, M., Slater, M.: Pertaub \& D, Razzaque, S, The responses of people to virtual humans in an immersive virtual environment. Presence 14(1), 104-116 (2005)

14. Goodrich, M.A., Schultz, A.C.: Human-robot interaction: a survey. Foundations and Trends in Human-Computer Interaction 1(3), 203-275 (2007)

15. De Graaf, M., Ben Allouch, S.: Exploring influencing variables for the acceptance of social robots. Robotics and Autonomous Systems 61(12), 1476-1486 (2013) 
16. Gunhee Kim, W.C., Kim, K.-R., Kim, M., Han, S., Shinn, R.H.: The autonomous tourguide robot Jinny. In: Proceedings from IEEE/Robotics Society of Japan International Conference on Intelligent Robots and Systems. IEEE (2004)

17. Halpern, D., Katz, J.E.: Close but not stuck: understanding social distance in human-robot interaction through a computer mediation approach. Intervalla 1 (2013)

18. Haraway, D.J.: A manifesto for cyborgs: Science, technology and socialist feminism in the. Socialist Review 80(15), 65-107 (1985)

19. Harlow, H.F.: The nature of love. American Psychologist 13(12), 673-685 (1958)

20. Knapp, M.L., Hall, J.A., Horgan, T.G.: Nonverbal communication in human interaction, 8th edn. (2013)

21. Lackey, S., Barber, D., Reinerman, L., Badler, N.I., Hudson, I.: Defining Next-Generation Multi-Modal Communication in Human Robot Interaction. In: Proceedings of the 55th Annual Meeting of the Human Factors and Ergonomics Society, HFES (2011)

22. Lackey, S., Barber, D., Reinerman-Jones, L.E., Ortiz, E., Fanfarelli, J.: Human Robot Communication. In: Hale, K., Staney, K. (eds.) Handbook of Virtual Environments, 2nd edn. (2013)

23. Lincoln, P., Welch, G., Nashel, A., State, A., Ilie, A., Fuchs, H.: Animatronic shader lamps avatars. Virtual Reality 15(2-3), 225-238 (2010)

24. McFarland, H.F., Barkhof, F., Antel, J., Miller, D.H.: The role of MRI as a surrogate outcome measure in multiple sclerosis. Multiple Sclerosis 8(40) (2002)

25. Mumm, J., Mutlu, B.: Human-robot proxemics: physical and psychological distancing in human-robot interaction. In: Proceedings of the 6th International Conference on Humanrobot Interaction, pp. 331-338. ACM (2011)

26. Nomura, T., Takagi, S.: Exploring effects of educational backgrounds and gender in human-robot interaction. In: Proceedings of 2011 International Conference on User Science and Engineering (i-USEr), pp. 24-29. IEEE (2011)

27. Oatley, K., Johnson-Laird, P.N.: Cognitive approaches to emotions. Trends in Cognitive Sciences (2014), Advance online publication doi: 10.1016/j.tics.2013.12.004

28. Reeves, B., Nass, C.: The media equation. Cambridge University Press, Cambridge (1996)

29. Reinerman-Jones, L.E., Sollins, B., Hudson, I.: Evaluating Human-Robot Implicit Communication using Psychophysiology. In: Proceedings of the Annual Meeting of AUVSI Unmanned Systems North America (2013)

30. Salvini, P., Laschi, C., Dario, P.: Design for acceptability: improving robots' coexistence in human society. International Journal of Social Robotics 2(4), 451-460 (2010)

31. Schermerhorn, P., Scheutz, M., Crowell, C.: Robot social presence and gender: do females view robots differently than males. In: Proceedings of the 3rd ACM International Conference on Human-Robot Interaction, pp. 263-270 (2008)

32. Shaw-Garlock, G.: Looking forward to sociable robots. International Journal of Social Robotics 1(3), 249-260 (2009)

33. Sidner, C.L., Kidd, C.D., Lee, C., Lesh, N.: Where to look: a study of human-robot engagement. In: Proceedings of the 9th International Conference on Intelligent User Interfaces, pp. 78-84. ACM (2004)

34. Stevens, L.E., Fiske, S.T.: Motivation and cognition in social life: A social survival perspective. Social Cognition 13(3), 189-214 (1995)

35. Watzlawick, P., Beavin, J.H., Jackson, D.D.: Pragmatics of Human Communication. W.W. Norton, New York (1967)

36. Waytz, A.: Social connection and seeing human. In: Dewall, C.N. (ed.) The Oxford Handbook of Social Exclusion. Oxford University Press, New York (2013) 
37. Welch, G.: Physical-virtual humans: challenges and opportunities. In: Proceedings from ISUVR 2012: International Symposium on Ubiquitous Virtual Reality (2012)

38. West, R., Turner, L.H.: Understanding interpersonal communication: Making choices in changing times, vol. 2. Wadsworth Cengage Learning, Boston (2009)

39. Witmer, B., Singer, M.: Measuring presence in virtual environments: A presence questionnaire. Presence 7(3), 225-240 (1998)

40. Yanco, H.A., Drury, J.L., Scholtz, J.: Beyond usability evaluation: Analysis of humanrobot interaction at a major robotics competition. Human-Computer Interaction 19(1-2), 117-149 (2004)

41. Yamashita, J., Kuzuoka, H., Fujimon, C., Hirose, M.: Tangible avatar and tangible earth: a novel interface for astronomy education. Proceedings from CHI 2007, Extended Abstracts on Human Factors in Computing Systems. ACM (2007)

42. Yuan, Y.C., Fulk, J., Monge, P.R., Contractor, N.: Expertise directory development, shared task interdependence, and strength of communication network ties as multilevel predictors of expertise exchange in transactive memory work groups. Communication Research $37(1), 20-47$ (2010) 